\title{
Testing Spatial Detection and Light Sensitivity in Homonymous Hemiano- pia by Rarebit and Conventional Automated Perimetry
}

\author{
Carlo Aleci ${ }^{*}$ and Tiziana Usai
}

Department of Ophthalmology, Gradenigo Hospital, C.so R.Margherita 8, 10153 Turin, Italy

\begin{abstract}
Purpose of the study was to compare Rarebit Perimetry (RP) with Standard Automatic Perimetry (SAP) in patients with ischemic retrochiasmal lesions. Seventeen patients with homonymous hemianopia underwent SAP and RP. Correlation between the two techniques was evaluated, congruent defects were quantified in the spared hemifield and residual vision areas were investigated in the blind hemifield. A strong correlation was found between SAP and RP. In the spared hemifield, RP detected congruent defects in a higher percentage of cases. Residual detection function has been found at RP in 69,2\% of perimetrically blind visual field quadrants as assessed by SAP. In conclusion, RP appears to be useful to assess visual function in hemianopic patients. As compared to SAP, it seems able to detect small congruent defects in a higher percentage of cases. Finally, a certain residual function seems to be present at RP in the blind regions of the visual field, however, for this finding further demonstrations are required.
\end{abstract}

Keywords: Hemianopia, rarebit perimetry, automated perimetry, congruent defect, residual visual function.

\section{INTRODUCTION}

Visual field defects are a main consequence of cerebrovascular accidents and cerebral tumours due to the wide representation of the visual pathway in the central nervous system [1].

It has been estimated that in about $20-30 \%$ of strokes visual system is involved to a certain degree [2,3].

Consequently, typical visual field defects that are hemianopia or quadrantanopia are frequently found in patients using Standard Automated Perimetry (SAP). In the last few years, although attempts have been made to find a more effective perimetric test in evaluating such neurophthalmological disorders, conventional perimetry remains the gold standard. In fact, neither Frequency Doubling Technology (FDT) [4-8], nor objective perimetry as multifocal VEP [9] or pupillometry [10] have proved to be more sensitive. Only Short Wavelength Automated Perimetry (SWAP) seems to be more sensitive than SAP in demyelinating or vascular diseases [11-13] but, as already stated [14], SWAP usefulness is strongly limited.

It is important to stress that hemianopic defects as tested by SAP are referred to as light sensitivity. Nonetheless, there is evidence that other visual functions [15-21] could result in a different degree or extent of damage in the affected hemifield or after geniculostriate differentiation.

Rarebit Perimetry (RP) is a new perimetric test developed by Frisen [22-24] which seems to be promising in the detection of visual field damage in patients with neurological diseases. It differs from other perimetric techniques as it measures the frequency in detecting minimum informational

*Address correspondence to this author at the Department of Ophthalmology, Gradenigo Hospital, C.so R.Margherita 8, 10153 Turin, Italy; Tel: +39 340000 3523; Fax: +39 (0)11 8151274; E-mail: carlo.aleci@h-gradenigo.it stimuli across the visual field, that is microdots displayed for a brief time and at a suprathreshold level. Under this basis, rather than testing light sensibility we can assume it measures a different function that is visual detection over space $[22,25,26]$.

In this study spatial visual detection has been evaluated in patients affected by homonymous hemianopia/quadrantanopia by means of RP. Quadrantanopias were considered as incomplete hemianopic defects, so both types of patterns were combined and analysed as a single group. Results have been correlated with SAP in order to estimate if RP can be more informative in discovering minimal defects in the spared hemifield and in assessing functional differences in the compromised hemifield.

\section{MATERIALS AND METHODS}

The rarebit technique has been described in detail in a previous article by Frisén [22]. It aims to evaluate the neuroretinal matrix looking for gaps in receptive field spatial continuity. Rather than spots of light on a white background, as it is used in conventional perimetry, visual stimuli employed are two high contrast white microdots (dot size: $50 \%$ of the minimum angle of resolution, MAR) on a dark background of a PC liquid crystal screen. Dot contrast does not change during the examination while dot size increases with eccentricity, so as to keep its MAR constant in every locus in the visual field. Patients are presented two stimuli separated by $4^{\circ}$ are displayed simultaneously at random positions for a brief time (200 ms).

Examination was performed in monocular conditions. The patient is required to look at a fixation cross and to indicate if no dots, one dot or both dots are perceived by not clicking, clicking or double-clicking a mouse button. Under these conditions and according to the test program, we have chosen 24 rectangular areas $\left(6^{\circ} \times 8^{\circ}\right.$ wide in the centre and 
$6^{\circ} \times 14^{\circ}$ wide in periphery) covering an overall visual field extended $30^{\circ}$ horizontally and $20^{\circ}$ vertically from the fixation point. In each area, dot detection is repeatedly evaluated $n$ times (or passes), until the technician interrupts the test. For our experiments we adopted ten passes which strike a balance between high degree accuracy and examination duration.

The main characteristic of rarebit technique is its probabilistic approach. Rather than thresholding visual function, the test expresses functional integrity of visual field in every locus as a percentage of hit rates over the total number of presented stimuli.

The program provides a global index, Mean Hit Rate, expressed as percentage (MHR, \pm SD) and obtained by averaging single results from every locus except the two areas next to the blind spot. The lower normal limit for MHR has been set at $92.7 \%$ [23]. In SAP correspondent index it may be regarded as Mean Sensitivity (MS).

17 patients (12 males, 5 females) suffering from homonymous hemianopia or quadrantanopia as diagnosed at SAP and resulting from post-stroke cerebral lesion as documented at CT and MNR were recruited. Of these patients, 10 $(58,8 \%)$ had an hemianopia and $7(41,2 \%)$ a quadrantanopia. The time of onset of neurological symptoms varied from 2 to 117 months (Table 1).

According to Wall et al. [4], a visual field defect was considered as hemianopic (or quadrantanopic) if the whole hemifield (or quadrant) was depressed with a sharp separation along the vertical meridian and without any other abnormal test location that would obscure the homonymous pattern.

Mean age was 62,3 years $( \pm 12,7$, range $34-80)$. Exclusion criteria were best corrected visual acuity (BCVA) < 0.7 , ametropia $> \pm 3$ diopters, ophthalmological diseases, intraocular pressure higher than $18 \mathrm{mmHg}(\mathrm{G})$ or inheritance for glaucoma, strabismus, amblyopia, diabetes, systemic therapies able to induce visual field defects, cognitive disability and poor cooperation. Every patient showed stable foveal fixation as tested by Cuppers' visuscope.

The Neuro-Ophthalmology Service of the Opthalmology Department of Gradenigo Hospital, Turin, recruited all subjects.

As a control group, 14 normal subjects (mean age $=57$ years, $\pm 10,85$, range $34-70$ ) were enrolled from the staff of the hospital. Exclusion criteria were the same as those of patients. Subjects with systemic hypertension or inheritance for cerebral vascular diseases were excluded. All control subjects had normal or corrected to normal visual acuity.

After obtaining informed consent, subjects underwent a complete ophthalmological examination (including slit lamp examination, retinoscopy, tonometry, visual acuity measurement, pupillary reflexes and ocular movement evaluation). Conventional automated perimetry was performed by means of an Octopus 1-2-3 perimeter (Haag-Streit, Bern,

Table 1. Demographic and Anamnestic Data of Recruited Patients. Subjects 15, 16 and 17 were Excluded for Low Reliability of their Examinations

\begin{tabular}{|c|c|c|c|c|c|c|}
\hline Patient & Sex & Age (y) & Months Since Onset & Refraction (sfer. equival.) $(\mathrm{R} / \mathrm{L})$ & Localization & Perim.Pattern \\
\hline 1 & $\mathrm{f}$ & 74 & 2 & $4,00 / 4,25$ & temporal-occipital $\mathrm{R}$ & LEFT HEMI \\
\hline 3 & $\mathrm{f}$ & 72 & 11 & $1,25 / 1,25$ & occipital R & LEFT HEMI \\
\hline 4 & $\mathrm{~m}$ & 64 & 25 & $1,00 / 0,75$ & occipital L & RIGHT HEMI \\
\hline 6 & $\mathrm{f}$ & 68 & 9 & $1,25 / 1,00$ & frontal+par+tem+occipital $\mathrm{R}$ & LEFT HEMI \\
\hline 7 & $\mathrm{~m}$ & 34 & 60 & $-1,75 /-1,75$ & occipital R & LEFT HEMI \\
\hline 8 & $\mathrm{~m}$ & 56 & 117 & $-1,50 /-2,00$ & vertebro-basilar $\mathrm{R}$ & LEFT HEMI \\
\hline 9 & $\mathrm{~m}$ & 75 & 32 & $-2,00 /-1,50$ & temporal-occipital $\mathrm{R}$ & LEFT HEMI \\
\hline 13 & $\mathrm{f}$ & 63 & 50 & $1,50 / 1,50$ & occipital L & RIGHT SUP QUAD \\
\hline 14 & $\mathrm{f}$ & 39 & 11 & $-3,50 /-5,00$ & Not specific & LEFT INF QUAD \\
\hline 15 & $\mathrm{~m}$ & 66 & 4 & $1,50 / 1,00$ & frontal+temporal L & RIGHT INF QUAD \\
\hline 16 & $\mathrm{~m}$ & 51 & 18 & $-1,25 /-1,25$ & parietal+occipital L & RIGHT INF QUAD \\
\hline 17 & $\mathrm{~m}$ & 71 & 14 & $2,25 / 2,25$ & occipital R & LEFT SUP QUAD \\
\hline MEAN & & 62,3 & 30,1 & & & \\
\hline$S D$ & & 12,7 & 31,7 & & & \\
\hline$R A N G E$ & & $34-80$ & $2-117$ & & & \\
\hline
\end{tabular}


Switzerland), program $32 \mathrm{x}$, which analyses central $30^{\circ}$ visual field (76 locations on a $6^{\circ}$-spaced grid) with a Goldmann size III stimulus $\left(0,43^{\circ}\right.$ wide $)$.

Rarebit perimetry (version 4) was performed first at a distance of $50 \mathrm{~cm}$ to test peripheral loci ("outer" modality), then at 1 meter to test central loci ("inner" modality). "Outer" and "inner" modalities are not modification of the original methodology, which automatically provides to adjust the size of the targets and their separations appropriate to the two viewing distances employed. All patients were acquainted in conventional perimetry while rarebit examination was preceded by a short training course (10 minutes) to increase confidence with the new technique. Calibration of the instrument was performed before every trial.

Sessions took place in a dimmed room (average illumination: 1 lux). The patients head was kept fixed by a chin rest at a distance of 0,5 meters to test the 20 peripheral areas and later at 1 meter to evaluate the remaining 4 inner locations. Adequate lens was used for the examination distance and eventually to correct refractive defects.

The same operator, skilled in psychophysical procedures, tested eyes in random order.

Reliable criteria for conventional perimetry were: reliability factor $(\mathrm{RF})<25 \%$ and false positive answer less than $10 \%$, while for Rarebit: error statistic value $\leq 4$ (for 10 passes).

\section{The study considered:}

for SAP: MS, MS in the affected and spared hemifield. To investigate congruous defects in the spared hemifield, the percentage of significant depressed points (or clusters) $(\mathrm{p}<5 \%)$ with corresponding defect in the spared hemifield of the fellow eye were calculated in the Comparison map.

In this case the adopted arbitrary criterion was:

a) Exact spatial correspondence (that is absolute congruence) of the two points (or clusters) in the two eyes with a difference $\leq 4 \mathrm{~dB}$, or

b) The same but with a difference $\leq 2 \mathrm{~dB}$.

These specific tolerance levels would correspond to the upper limit of light threshold fluctuation as referred in normal subjects $[27,28]$.

We will refer to these values as SAP Congruence Levels $\left(\mathrm{CL}_{\mathrm{SAP} 4}, \mathrm{CL}_{\mathrm{SAP} 2}\right)$.

To minimize bias due to fixation loss, the six points around the blind spot were not considered.

For RP: MHR (excluding the two areas closest to the blind spot), MHR in the affected and spared hemifield. To investigate congruous defects in the spared hemifield the percentage of areas with Miss Rate $\geq 5 \%$ with corresponding defective areas in the spared hemifield of the fellow eye has been calculated in the RP Miss Rate map.

In this case the adopted arbitrary criterion was:

a) Exact spatial correspondence of the two areas in the two eyes with a Miss Rate difference $\leq 10 \%$, or b) The same but with a Miss Rate difference $\leq 5 \%$.

These values are close to the upper limit of Short Term and Long Term Fluctuation in Rarebit Perimetry in a global and point-to point analysis [29].

We will refer to these values as RP Congruence Levels $\left(\mathrm{CL}_{\mathrm{RP} 10}, \mathrm{CL}_{\mathrm{RPS}}\right)$.

In these cases the two areas corresponding to the blind spot were not considered in the spared hemifield.

To assess functional differences between light sensitivity and spatial detection in the affected hemifield, areas with residual function detected at RP and MHR was evaluated in each perimetrically blind quadrant as tested by SAP. In this case quadrantanopias have been excluded and only hemianopias (10 patients) were examined.

A quadrant was considered as perimetrically blind if one of these two conditions were satisfied at SAP:

a) Absolute scotoma in each of the nineteen locations of the quadrant, or

b) Deep depression (sensibility $\leq 5 \mathrm{~dB}$ ) involving $\leq 4$ locations along the edge of the total length of vertical meridian with abolished sensitivity in the remaining points of the edge and in the rest of the affected quadrant. In this case, the residual level of sensibility along the vertical meridian has been ascribed to fixation shift.

However, to avoid any influence by fixation instability or fixation losses in hemianopic visual fields, in the rarebit map the two indexes were evaluated only on the three more peripheral areas (located from $10^{\circ}$ to $30^{\circ}$ of eccentricity), excluding the three remaining locations nearest to the vertical meridian.

When tested by RP, an area was regarded as having a residual function if its Hit Rate value was $\geq 15 \%$ in a first estimation or as a second paradigm, $\geq 10 \%$. For these two cases, two different analyses were done.

Correlation between MS and MHR in the whole field and between hemifields was analysed by Pearson's correlation analysis. Kolmogorow-Smirnow test was used to test for normality. Paired t-test was used to compare examination time and differences between patients and control subjects. Mann-Withney test was employed to analyse differences between results in both examinations. Statistical significance level was set for $p$ value $\leq 0,05$.

After mean values, in brackets is given standard deviation.

We certify that the research followed the tenets of the Declaration of Helsinki, that informed consent was obtained from the subjects after explanation of the nature and possible consequences of the study and that all applicable institutional and governmental regulations concerning the ethical use of human volunteers were followed.

\section{RESULTS}

Of the 17 recruited patients, 14 completed the experiment while 3 were excluded for the high rate of errors in one or 
both tests. None of the 14 normal subjects demonstrated any visual field defect at SAP.

Mean MS in the control group and in the group of patients was $27,7 \mathrm{~dB}( \pm 0,81)$ and $14,7 \mathrm{~dB}( \pm 3,60)$, while MHR was $96,8 \%( \pm 1,75 \%)$ and $52,2 \%( \pm 12,30 \%)$ (Difference: $\mathrm{p} \leq 0,01$ in both cases). In both groups RP examination time was significantly lower compared to SAP examination time performed by Octopus 1-2-3 (in controls: RP: 568,2 sec
$( \pm 33,96)$, SAP: $1211,7 \mathrm{sec}( \pm 55,82)$; in patients: RP: 648,79 $\sec ( \pm 56,91)$, SAP: $1138,54 \mathrm{sec}( \pm 128,62), \mathrm{p} \leq 0,01)$. Topographical correspondence of damage was very high in the two maps (Fig. 1).

In Fig. (2) is shown SAP/RP correlation analysis. Regarding the whole field significant correlation was found between SAP MS and RP MHR ( $r=0,78, p<0,01)$ (Fig. 2, left).

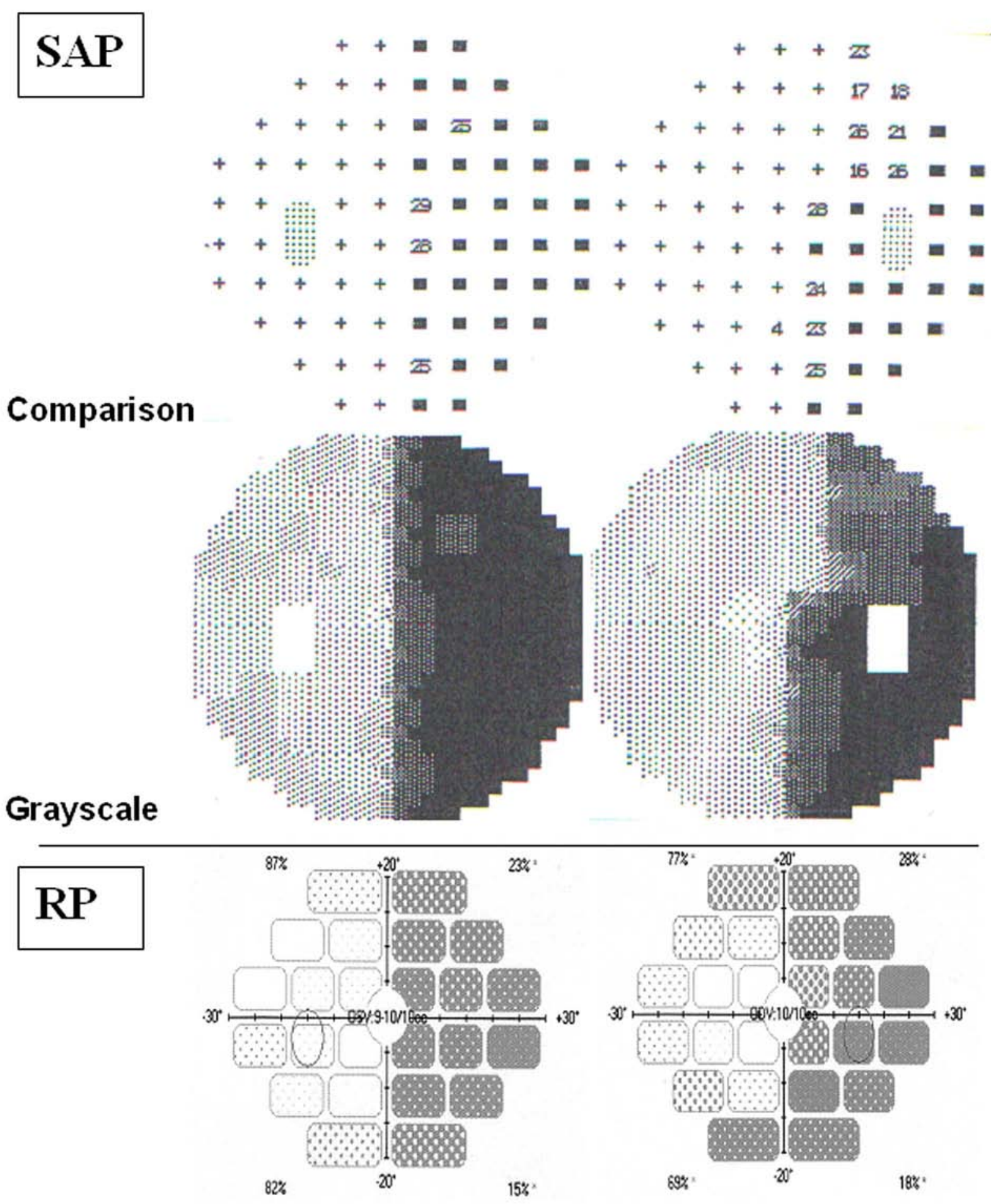

Fig. (1). Topographical correspondence between SAP and RP in a 64-year old patient with right homonymous hemianopia. 

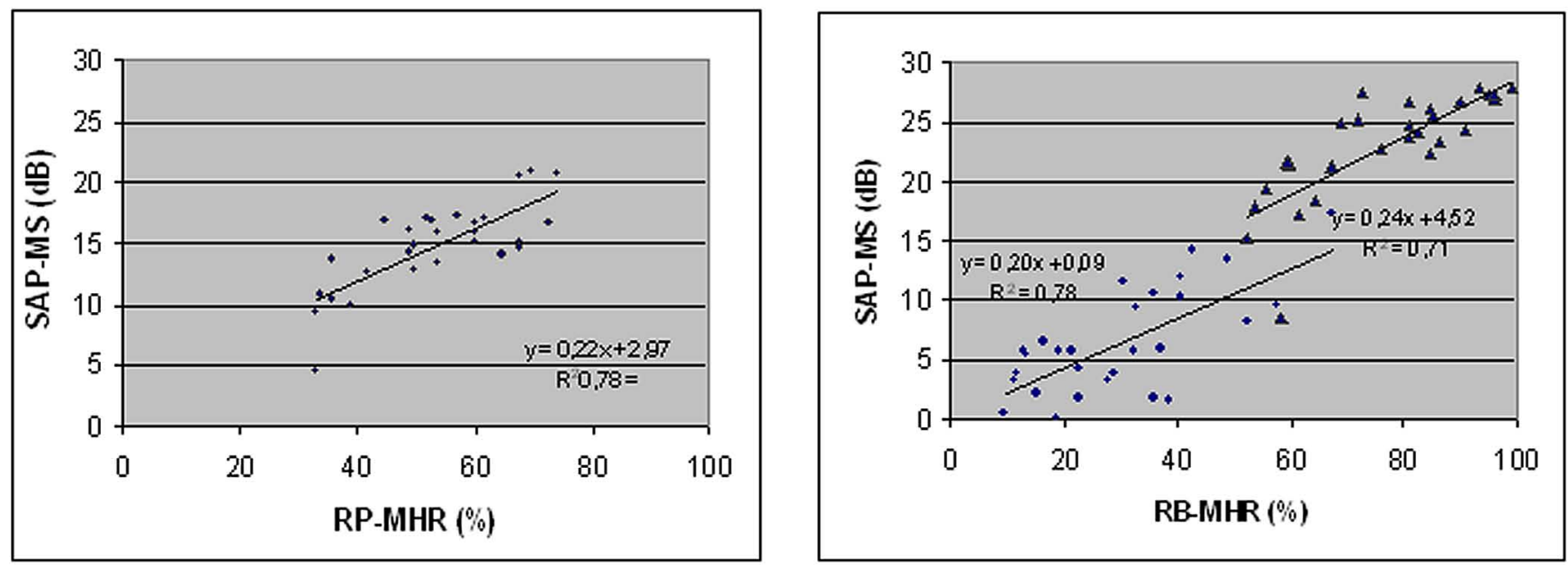

Fig. (2). Correlation between Standard Automated Perimetry Mean Sensitivity (SAP-MS) and Rarebit Perimetry Mean Hit Rate (RP-MHR) in patients affected by homonimous hemianopia and quadrantanopia. Left: whole field; Right: spared (circles) and affected (triangles) hemifields.

Correlation coefficient between RP MHR and SAP MS in the spared hemifield resulted to be the same as that related to the whole perimetric map $(\mathrm{r}=0,78, \mathrm{p}<0,01))$, while a slightly lower correlation was found in the affected hemifield $(\mathrm{r}=0,71, \mathrm{p}<0,01)$ (Fig. 2, right).

To quantify the congruence level of damage in the spared hemifields of both eyes, corresponding defective locations as a percentage have been calculated. It has been made for two values of defect in SAP and RP as stated previously. As shown in Table 2, percentages of defective points in the spared hemifield were higher with RP than with SAP, for both congruence levels. Compared to SAP, RP was able to find congruent defects in a higher number of patients, irrespective of the CL value. Considering each technique, there were no differences in the number of defective points/areas detected using the two CLs $(p>0,05)$.

To investigate residual visual function in the perimetrically blind region of the visual field the number of areas and MHR for each blind quadrant was evaluated, as specified in the methods section. Of the 40 quadrants obtained by SAP maps of our 10 hemianopic patients, 13 quadrants $(32,5 \%)$ were found to be perimetrically blind as tested by SAP according to our criteria. In 9 of these $(69,2 \%)$ some residual function was found when tested by RP with MHR cut-off set $\geq 10 \%$ and in $8(61,5 \%)$ with MHR cut-off set $\geq 15 \%$.

In the perimetrically blind quadrants, the total number of residual areas was 21 (1,6 area per quadrant) (cut-off $\geq 10 \%$ ) and 13 ( 1 area per quadrant) (cut-off $\geq 15 \%$ ).

No correlation was found between residual MHR and on- set time of accident (Spearman $\left.r_{s}=-0,05, p>0,05\right)$ or age of patients (Spearman $r_{s}=-0,15, p>0,05$ ).

\section{DISCUSSION}

It is well known that one of the major shortcomings of perimetry testing is its duration. An alternative technique to Standard Automated Perimetry, able to test rapidly visual field of patients affected by cerebral stroke is convenient, particularly considering their peculiar conditions, being often unable to perform a tiring full threshold examination. The Rarebit Perimetry has been demonstrated to be useful in ophthalmological practice [22-24, 30-36]. A recent study [37] has suggested it to be as effective as Humphrey standard automated perimetry in detecting homonymous hemianopia.

According to our results, rarebit technique could be a valid alternative in hemianopic patients considering its good correlation to Octopus Standard Automated Perimetry (taken as gold standard) and its short duration. Test-retest variability (in normal subjects) seems not to be greater than that found in SAP as found by Frisén [22] and Corallo [29].

Once the presence of hemianopia has been assessed, attention in ophthalmological follow-up is focused in particular on the spared hemifield to survey the onset of new defects. In this respect, results we obtained suggest a higher sensitivity in detecting localized congruent defects for RP as compared to conventional perimetry. As already suggested [22], a possible explanation can rely on the strong reduction of informational content in the RP target, as compared to that used in conventional perimetry and in the lower light scattering from microdot stimuli so that defective areas in the

Table 2. Congruence Levels Analysis in the Spared Hemifield. In Brackets is SD

\begin{tabular}{|c|c|c|c|}
\hline$C L_{S A P 4} ; C L_{R P 10}($ locations \%) & $12,85(13,89)$ & $33,57(18,65)$ & 0,003 \\
\hline hemifields with congruent defects for $C L_{S A P 4}$ and $C L_{R P 10}(\%)$ & 78,5 & 92,80 & ns \\
\hline hemifields with congruent defects for $C L_{S A P 2}$ and $C L_{R P 5}(\%)$ & 78,5 & 92,80 & $\mathrm{~ns}$ \\
\hline
\end{tabular}


spared region were not affected by stimuli projected to a neighbouring location.

It has been underlined in a previous study the problem of scattered abnormal depressed regions that may confound the typical hemianopic pattern [4]. Due to the microdot stimulation, a localized reduction in MHR can result as a consequence of physiological angioscotoma, without reflecting a real defect [38, 39].

In the blind regions of impaired visual fields, there is wide psychophysical (and electroretinographical [40]) evidence of the persistence of a little amount of visual sensibility. In particular some functions as motion perception [16$19,41]$, spatial vision detection $[1,20]$ or color $[18,21]$ and orientation discrimination [21] result to be partly spared or less affected than light sensibility. The case of the patient who is aware of his residual visual perception (i.e. a different condition than the so-called blindsight [41]) has been ascribed to activity of spared occipital cortical cells $[17,42]$ or optic fibres of the geniculo-striate visual pathway [43].

Indeed, the finding at RP of residual visual function in the perimetrically blind region (as assessed by SAP) supports this eventuality.

Caution must be used in this conclusion, for the possibility of a light scattering effect inducing an illusory perception in the blind region or, more probably, of a fixation shift affecting spatial localization of the perceived target [44-46]. Indeed a scattering effect seems to be less effective than in conventional perimetry due to the very small size of the RP stimuli. In recruited patients neither eccentric fixation nor macular plitting at SAP was found and fixation stability would be due to foveal sparing, covering a field extension of about 1,5 deg [46-48]. On the other hand, in rarebit perimetry it is impossible to detect and neutralize presentations occurring during a shift of fixation since a way for controlling this variable is not available. For this reason residual function analysis was performed only in those locations more than 10 deg apart from the midline, though this way a great amount of information across the visual field would inevitably be lost.

About this point, however, further confirmations are required, using at SAP a wider stimulus or an higher resolution grid (1 deg wide).

\section{CONCLUSION}

In conclusion, rarebit perimetry is a rapid and suitable perimetric technique in patients suffering from post-stroke visual field impairment. It appears to be more sensitive than SAP in detecting congruent defects in the spared hemifield. For these reasons it seems valid either as a screening or a follow-up test in patients with hemianopic and quadrantanopic defects. Moreover, if our data are confirmed by further research, RP could be employed to explore residual visual function in the blind regions of the visual field.

\section{REFERENCES}

[1] Gilhotra JS, Mitchell P, Healey PR, Cumming RG, Currie J. Homonymous visual field defects and stroke in an older population. Stroke 2002; 33: 2417-20.

[2] Huber A. Homonymous hemianopia. Neuroophthalmol 1992; 12: $351-66$.
Smith JL. Homonymous hemianopia, a review of one hundred cases. Am J Ophthalmol 1962; 54: 616-23.

Wall M, Neahring RK, Woodward KR. Sensitivity and specificity of frequency doubling perimetry in neuro-ophthalmic disorders: a comparison with conventional automated perimetry. Invest Ophthalmol Vis Sci 2002; 43: 1277-83.

Cerio-Ramsden CD, Munoz-Negrete FJ, Moro JGM, Rebolleda G. Frequency doubling Perimetry in retrochiasmal disorders. Arch Soc Esp Oftalmol 2003; 78(3): 143-9.

Thomams D, Thomas R, Muliyil JP, George R. Role of frequency doubling perimetry in detecting neuro-ophthalmic visual field defects. Am J Ophthalmol 2001; 131: 734-41.

Noval S, Contreras I, Rebolleda G, Munoz-Negrete FJ, Ruiz De Zarate B. A comparison between Humphrey and frequency doubling perimetry for chiasmal visual field defects. Eur J Ophthalmol 2005; 15: 739-45.

Neahring RK, Wall M, Withrow K. Sensitivity and specificity of frequency doubling perimetry in neuro-ophthalmic disorders. Invest Ophthalmol Vis Sci Suppl 1997; 38: S390.

Wall M. What's new in Perimetry. J Neuroophthalmol 2004; 24 : 46-55.

Rajan MS, Bremner FD, Riordan-Eva P. Pupilloperimetry in the diagnosis of functional visual field loss. J Royal Soc Med 2002; 95 : 498-500.

Keltner J, Johnson CA. Short-wavelenght automated perimetry in neuro-ophthalmological disorders. Arch Ophthalmol 1995; 113: 475-81.

Fushimoto N, Adachi-Usami E. Use of blue-on-yellow perimetry to demonstrate quadrantanopia in multiple sclerosis. Arch Ophthalmol 1998; 116: 828-9.

Fushimoto N, Kubota M, Saeki N, Adachi-Usami E. Use of blueon-yellow perimetry for detection of sectoranopia. Eye 2004; 18: 338-41.

Wild JM. Short wavelength automated perimetry. Acta Ophthalmol Scand 2001; 79: 546-59.

Barbur JL, Ruddock KH, Waterfield VA. Human visual response in the absence of geniculo-calcarine projection. Brain 1980; 103: 90528.

Ptito A, Lepore F, Ptito M, Lassonde M. Target detection and movement discrimination in the blind field of hemispherectomized patients. Brain 1991; 114: 497-512.

Intriligator JM, Xie R, Barton JJSS. Blindsight Modulation of Motion Perception. J Cogn Neurosci 2002; 14: 1174-83.

Morland AB, Jones SR, Finlay AL, Deyzac, Le S, Kemp S. Visual perception of motion, luminance and colour in a human hemianope. Brain 1999; 122: 1183-98.

Morland AB, Le S, Carrol E, Hoffmann MB, Pambakian A. The role of spared calcarine cortex and lateral occipital cortex in the responses of human hemianopes to visual motion. J Cogn Neurosci 2004; 16: 204-18.

Perenin MT, Jeannerod M. Visual function within the hemianopic field following early cerebral hemidecortication in man-I. Spatial localisation. Neuropsychology 1978; 16: 1-13.

Boyer JL, Harrison S, Ro T. Unconscious processing of orientation and colour without primary visual cortex. Neuroscience 2005; 102: 16875-9.

Frisén L. New sensitive window on abnormal spatial vision:rarebit probing. Vis Res 2002; 42: 1931-9.

Frisén L. Spatial vision in visually asymptomatic subjects at high risk for multiple sclerosis. J Neurol Neurosurg Psychiatry 2003; 74: 1145-7.

Frisén L. Vigabartin-associated loss of vision: rarebit perimetry illuminates the dose-damage relationship. Acta Ophthalmol Scand 2004; 82: 54-8.

Parker AJ, Newsome WT. Sense and the single neuron: probing the physiology of perception. Ann Rev Neurosci 1988; 21: 227-77.

Westheimer G. Location and line orientation as distinguishable primitives in spatial vision. Proc Royal Soc Lond 1996; 263:503-8. Bebie H, Fankhauser F, Spahr J. Static perimetry: Accuracy and Fluctuations. Acta Ophthalmol Scand 1976; 54: 339-49.

Fankhauser F, Bebie H. Threshold fluctuations, interpolations and spatial resolution in perimetry. Doc Ophthalmol Proc Ser 1979; 19: 295-309.

Corallo G, Bagnis A, Scotto R, Iester M, Calabria G. Rarebit perimetry in soggetti sani: effetto apprendimento e fluttuazione a breve e lungo termine. Ottica Fisiopat 2006; XI: 139-46. 
[30] Martin L, Wanger P. New perimetric techniques. A comparison between rarebit and FDT perimetry in normal subjects and glaucoma patients. J Glaucoma 2004; 13: 278-82.

[31] Brusini P, Salvetat ML, Parisi L, Zeppieri M. Probing glaucoma visual damage by rarebit perimetry. Br J Ophthalmol 2005; 89: 180-4.

[32] Nilsson M, von Wendt G, Martin L. Rarebit Foveal Test in diabetes. Acta Ophthalmol Scand 2006; 84: 567.

[33] Nilsson M, Wanger P, Martin L. Perception of very small visual stimuli in the fovea: normative data for the rarebit fovea test. Clin Exp Optom 2006; 89: 81-5.

[34] Martin L. Rarebit and frequency-doubling technology perimetry in children and young adults. Acta Ophthalmol Scand 2005; 83: 6707.

[35] Jacobson L, Flodmark O, Martin L. Visual field defects in prematurely born patients with white matter damage of immaturity: a multiple-case study. Acta Ophthalmol Scand 2006; 84: 357-62.

[36] Martin L, Ley D, Marshal K, Hellström A. Visual function in young adults following intrauterine growth retardation. J Pediatr Ophthalmol Strabismus 2004; 41: 212-8.

[37] Gedik D, Akman A, Akova Y. Efficiency of rarebit perimetry in the evaluation of homonymous hemianopia in stroke patients. Br J Ophthalmol Published Online first: 14 February 2007. doi:10.1136/bjo.2006.112607.

[38] Schiefer V, Benda N, Dietrich TJ, Selig B, Hofmann C, Schiller J. Angioscotomata detection with fundus-oriented perimetry: a study with dark and bright stimuli of different sizes. Vis Res 1999; 39: 1897-909.

[39] Adams DL, Horton JC. The representation of retinal blood vessel in primate striate cortex. J Neurosci 2003; 231: 5984-97.

[40] Azzopardi P, King SM, Cowey A. Pattern electroretinograms after cerebral hemisferectomy. Brain 2001; 124: 1228-40.

[41] Stoerig P, Cowey A. Blindsight in man and monkey. Brain 1997; 120: 535-59.

[42] Schärli H, Harman AM, Hogben JH. Residual vision in a subject with damaged visual cortex. J Cogn Neurosci 1999; 11: 502-10.

[43] Schärli H, Harman AM, Hogben JH. Blindsight in subjects with homonymous visual field defects. J Cogn Neurosci 1999; 11: 5266.

[44] Bischoff P, Lang J, Huber A. Macular sparing as a perimetric artifact. Am J Ophthalmol 1995; 119: 72-80.

[45] Jamara RJ, Van De Velde F, Peli E. Scanning eye movements in homonymous hemianopia documented by scanning laser ophthalmoscope retinal perimetry. Optom Vis Sci 2003; 80: 495-504.

[46] Trauzettel-Klosinski S, Jens R. The vertical field border in hemianopia and its significance for fixation and reading. Invest Ophthalmol Vis Sci 1998; 39: 2177-86.

[47] Reinhard J, Trauzettel-Klosinski S. Nasotemporal overlap of retinal ganglion cells in humans:a functional study. Invest Ophthalmol Vis Sci 2003; 44: 1568-72.

[48] Bunt A, Minckler DS, Johanson GW. Demonstration of bilateral projection of the central retina of the monkey with horseradish peroxidase neuronography. J Comp Neurol 1977; 171: 619-30.

This is an open access article licensed under the terms of the Creative Commons Attribution Non-Commercial License (http://creativecommons.org/licenses/bync/3.0/) which permits unrestricted, non-commercial use, distribution and reproduction in any medium, provided the work is properly cited. 